\title{
A Deep Learning Approach for Robotic Arm Control using Brain-Computer Interface
}

\author{
John Sahaya Rani Alex ${ }^{\text {b* }}$,Md Amaan Haque ${ }^{\mathrm{a}}$, Anuj Anand ${ }^{\mathrm{a}}$, Anmol Gautam ${ }^{\mathrm{a}}$, Nithya Venkatesan ${ }^{\mathrm{a}}$ \\ ${ }^{a}$ School of Electrical Engineering, Vellore Institute of Technology, 600127, Chennai, India \\ $\mathrm{b}^{*}$ Division of Healthcare Advancement,Innovation and Research, Vellore Institute of Technology, \\ 600127, Chennai, India
}

Received: May 25, 2020. Revised: September 15, 2020. Accepted: September 21, 2020. Published: September $23,2020$.

\begin{abstract}
Brain-Computer Interface (BCI) is a technology that enables a human to communicate with an external stratagem to achieve the desired result. This paper presents a Motor Imagery (MI) - Electroencephalography (EEG) signal based robotic hand movements of lifting and dropping of an external robotic arm. The MI-EEG signals were extracted using a 3-channel electrode system with the AD8232 amplifier. The electrodes were placed on three locations, namely, C3, C4, and right mastoid. Signal processing methods namely, Butterworth filter and Sym-9 Wavelet Packet Decomposition (WPD) were applied on the extracted EEG signals to de-noise the raw EEG signal. Statistical features like entropy, variance, standard deviation, covariance, and spectral centroid were extracted from the de-noised signals. The statistical features were then applied to train a Multi-Layer Perceptron (MLP) Deep Neural Network (DNN) to classify the hand movement into two classes; 'No Hand Movement' and 'Hand Movement'. The resultant k-fold cross-validated accuracy achieved was $85.41 \%$ and other classification metrics, such as precision, recall sensitivity, specificity, and F1 Score were also calculated. The trained model was interfaced with Arduino to move the robotic arm according to the class predicted by the DNN model in a real-time environment. The proposed end to end low-cost deep learning framework provides a substantial improvement in real-time BCI.
\end{abstract}

\section{Keywords-MI-EEG, BCI, DNN, Robotic Arm.}

\section{INTRODUCTION}

$\mathrm{T}$ HE brain is the center of all the activity in the human body and is part of the nervous system. Brain-Computer Interface (BCI) is a communication system in which messages or commands that an individual generates via brain are transferred to a computer and subsequently associated with use to control an external device [1]. BCIs are mainly used for managing and regulating movements as seen in the case of a motorized wheelchair, prosthetic limb, restoring mobility in paralyzed limbs by electrically stimulating muscles, controlling home appliances, controlling a robotic car, playing computer games, decoding brain activity to reproduce movements in prosthetic arms, controlling elements in virtual reality, typing a message on a computer screen by concentrating on the display, etc. This list [1]-[6] is expanding rapidly and a substantiate amount of research is being carried out especially to help patients with motor impairments.

Brain activity can be monitored by recording and analyzing signals like Electroencephalography (EEG), functional NearInfrared Spectroscopy (fNIRS), functional Magnetic Resonance Imaging(fMRI) and Electrocorticography (ECoG) [7]. EEG, fMRI, and fNIRS are non-invasive methods and ECoG is an invasive method. Among these, the most widely used signal is the EEG signal to monitor the brain activity.

The electroencephalography (EEG) signal records the brain's electrical activity by capturing variations in voltage that occur from internal neural activity and provides partial information about the physiological state of the human body [8]. EEG signals are categorized into Delta, Theta, Alpha, Beta, and Gamma waves based on the frequency range and the brain activity [9]. Alpha waves are pulsing and its frequency variation is from 8 to $13 \mathrm{~Hz}$. The amplitude of the cortical potential is low. Alpha rhythm is mostly associated with occipital and parietal regions of the brain but its characteristics can be found in most parts of the brain. Alpha rhythm oscillates every time regardless of an awake or relaxed state. Variations in Beta waves are considerably higher than the other waveforms and it records predominantly in the temporal and frontal lobe of the brain. It oscillates throughout the mental activity and is related to basic cognitive and motor activity. Beta wave frequency ranges from 13 to $30 \mathrm{~Hz}$. Gamma waves measure the quickest brainwave frequency and its frequency variation is from 31 to $100 \mathrm{~Hz}$. With the help of advanced digital signal processing techniques, measurement of these waves assist in diagnosing the brain's activity. Therefore its used as a utility in many assistive technologies and to diagnose brain/neurological disorders.

BCI interface has four functional blocks, the first being acquisition of brain signals, which includes a selection of sensors, placement of sensors [10], and humans' mental cooperation towards that. The second step is the processing of the signals which includes de-noising [11], [12], removing artefacts [13], signal enhancement [14] and feature extraction [3],[4],[9]. After the signal acquisition and feature extraction, depending upon the application, classifiers are trained with the handcrafted features. The final step is connecting to the external device.

In the first step, while recording the EEG signal, artefacts such as eye blinking [15] and power line interference are also 
mixed in the signal. To remove the artefacts, Butterworth filter [13] and wavelet transform [14] is applied. The feature extraction includes the usage of typical signal processing methods to extract the traditional features such as energy, power spectrum, Power Spectral Density (PSD), and statistical features [3],[16]-[18]. Discrete Wavelet Transform (DWT) based feature extraction methods are preferred [14],[17]-[19] because of its time-spatial domain analysis and de-noising ability over the Fourier Transform (FT). DWT filter bandwidths are non-linear. To have linear bandwidths, Wavelet Packet Decomposition (WPD) [11], [20]-[21] is widely useful as the feature extraction methods. Some researchers took advantage of combining both FT and DWT [22] in the feature extraction process. Another method of extracting time-frequency information other than DWT is Hilbert-Huang Transform (HHT) [15], [23].

Sometimes feature engineering is done to get handcrafted features. Features such as fractal dimension [24] and fuzzy wavelet packet [20] are extracted from the EEG signal. Recently, Convolutional Neural Networks (CNN) are used to generate features [25] from the EEG signal automatically without handcrafting them.

EEG signal is a high dimensional signal in nature, and thus increases the computational complexity of the classifier it goes with. To reduce the high dimension, dimensionality reduction techniques such as Principal Component Analysis (PCA) [4],[12],[18], Independent Component Analysis(ICA) [18] and Linear Discriminative Analysis(LDA) [13],[14],[21] are widely employed.

Typical pattern recognition classifiers are widely employed to classify the EEG signal. To mention a few, They are Hidden Markov Models [HMM] [17], Support Vector Machine [SVM] $[13,20]$, Artificial Neural Networks[ANN] [5,7,10] and Machine Learning[ML] algorithms [10]. Recently, deep learning models CNN [26, 27] and Long Short Term Memory (LSTM) [28] networks are employed to improve the efficacy of the EEG signal classifier. The convolutional neural network architecture employed in paper [27] achieved an accuracy of $71 / 82 \%(+4.2 \%)$ with a multichannel EEG signal as 2D image input. The accuracy got much improved when the convolution network was employed using the transfer learning technique [26]. The pre-trained model AlexNet is adapted for the classification of EEG signals through transfer learning [29]. Also, ResNet is adapted for MI EEG classification [26]. However, these networks improved the accuracy of the classification at the cost of huge computational complexity

Although a lot of research has been carried out to improve the intra-subject accuracy and inter-subject accuracy of the BCI task, accuracy is still less than $80 \%$. Therefore, a simplified innovative end to end methodology is necessary for further improvement.

There are few challenges in accomplishing the end to end BCI task.

- Placement of electrodes on the scalp

- The effectiveness of the ionic current flow varies from subject to subject

- EEG signal is low SNR
- The BCI task is application dependent; non-availability of standard data set for evaluating the model.

- Design of an external device which provides feedback to the subject

- Finally the integration of all the above at a low cost with high efficacy.

All the above challenges leave a room for research in BCI. In this work, we aim to propose a customized end to end a lowcost robotic arm control framework. The end to end low-cost framework objective is achieved through multi-fold. The first step is to design of low-cost data acquisition EEG device. Next, hand-collecting data for our application and finally, design of low computational complexity deep learning model. Though there are BCI datasets available [30], for this particular task of arm movement, there is no motor imagery EEG data available. We have designed a 3-channel data acquisition embedded board to get the data from the subject, feature engineered on the preprocessed data, designed a deep learning model with acceptable accuracy for the BCI task, and finally integrated the output with a robotic arm. Overall, the proposed framework showed significant improvement as a low-cost solution to the research community. We shall explain the proposed framework in detail in the following sections.

\section{METHODS}

\section{A. Data Collection}

The acquisition of the EEG signal was done using a simple three-channel electrode [17]. These electrodes are placed on three locations namely, C3, C4, and right mastoid [31,32] on the scalp of the subjects. Arduino Uno with the AD8232 sensor kit is connected to these three electrodes. The 3-channel EEG signal is received by the AD8232 and fed to a computer through Arduino for further processing. PLX-DAQ is an add-on software in Microsoft Excel that enables smooth and direct data acquisition from a microcontroller, such as the Arduino, to compute for further processing.

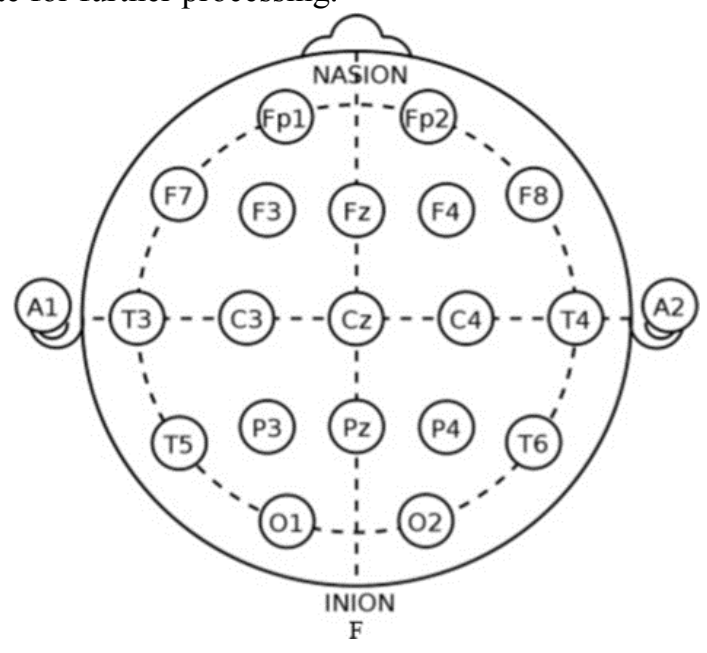

Fig. 1. International 10-20 Electrode Placement System

The International 10-20 Electrode Placement System, as 
shown in Fig. 1, was followed to place the electrodes in the proper positions to obtained clear signals during the data acquisition.

Table 1. Subject details

\begin{tabular}{cc}
\hline Subject name & Age \\
\hline HM_21_M & 21 \\
V_35_M & 35 \\
MK_37_M & 37 \\
CK_39_M. & 39 \\
H_59_M & 59 \\
\hline
\end{tabular}

Table 2 Data set

\begin{tabular}{cc}
\hline Data class & No of Samples \\
\hline Hand movement & 125 \\
\hline No hand movement & 125 \\
\hline
\end{tabular}

The EEG signals were recorded for a total of seven seconds from each subject and each class. For the class 'Hand Movement', subjects were asked to lift their hand at the end of the third second in the total seven-second interval. For the class 'No Hand Movement', the subjects did not lift or move their hand and were advised to remain calm. In total, data collections were taken from five different subjects belonging to different age groups ranging from twenty to sixty years, as listed in Table 1. For privacy, the subject name is coded here. The subjects were informed about this research and with their consent, the data acquisition from one of the subjects is depicted in Fig. 2. To keep the dataset balanced, for hand movement class 125 recordings were collected and for 'no hand movement' 125 recordings were collected. The hand-collected data set of 250 recordings were then split into $80 \%$ for training which are 202 samples and $20 \%$ for testing which are 48 samples.

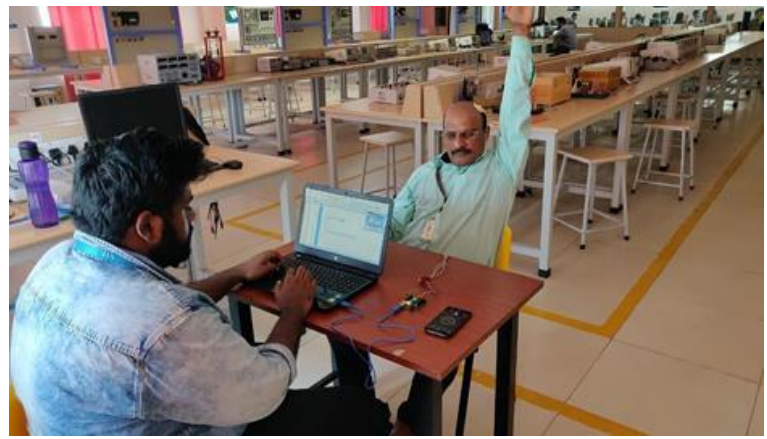

Fig. 2 Experimental data acquisition for hand movement

\section{B. Signal Pre-processing}

EEG Signals are always added with artefacts that introduce changes within the recorded cerebral activity. These artefacts could mimic the brain's psychological feature or pathological activity; these artefacts may additionally overlap with EEG frequency bands with amplitudes larger than the cortical signals. In general, many kinds of artefacts, as well as physiological and non-physiological artefacts, could corrupt the EEG features. Physiological artefacts originate from sources within the body, like the heart, eye, and muscles. In contrast, non-physiological artefacts are associated with surroundings and instrumentality. These artefacts lead to irregularities in the recorded EEG data. Therefore, signal pre-processing i.e. cleaning the data from unwanted signal need to be performed before using the data. Butterworth band-pass filter [13] is used to remove the unwanted waveband and in turn minimizes the number of features. It has an immediate impact on reducing the execution time and decreases the use of memory, which in turn boosts the system performance. Henceforth, the EEG signals are filtered with a bandpass filter with a frequency range of 12 to $30 \mathrm{~Hz}$, which is designed with a fourth-order Butterworth filter, to allow beta rhythm and to get rid of unwanted artefacts.

Wavelet Transform (WT) is a multi-resolution analysis compared to FT, which is a constant resolution. Therefore, WT is chosen for this research. Choosing a Mother Wavelet basis function is a challenge because not much in literature is mentioned. In this research work, the Sym9 wavelet transform, which exhibits a biorthogonal, near symmetric and orthogonal properties, is experimentally selected and employed to eliminate ocular physical object noise, eye blinking noise, and internal organ artefacts.

From the filtered, pre-processed EEG signal, fourteen statistical features are extracted namely Mean, Maximum Amplitude, Minimum Amplitude, Standard Deviation, Variance, RMS, Entropy, Kurtosis, Skewness, Percentile, Median, Covariance, Centroid, and Spectral Centroid.

\section{Deep Neural Network-Multi-Layer Perceptron (DNN- $M L P)$ Classifier}

A Deep Neural Network (DNN) with two hidden layers is shown in Fig. 3. DNNs are mathematical models that resemble the neural design of the brain and work together with interconnected neurons (nodes).

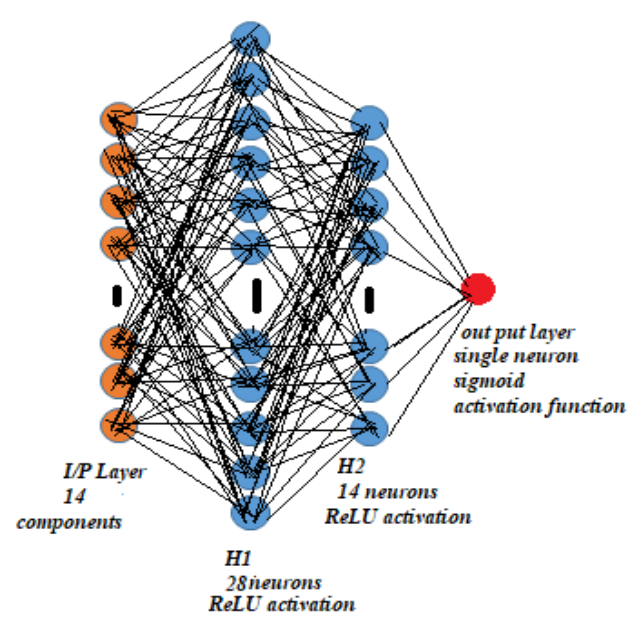

Fig. 3. Proposed Deep Neural Network architecture 
DNNs [33] are ANNs with two or more hidden layers. For instance, an ANN's perceptron contains input neurons just as the brain contains dendrites, similarly hidden neurons for brain neurons, output neurons for axons, and the connection between two neurons just as synapses in the brain. DNNs also work very similarly to how a brain does. The inputs are given through the input layer and processing is done by the neurons in the hidden layers present in between the input and output layers. It is the hidden neuron that decides whether to pass the signal further or not. The final result is predicted by the output layer and is then compared with the actual result. Based on this comparison, the cost function and the error rate are calculated. This information is sent back to the neural network and the weights of the neurons are adjusted accordingly to achieve the lowest cost function.

\section{Robotic Arm Control}

A 3-D printed robotic arm has been purchased [34] and used for mimicking the robotic arm. The arm which has two degrees of freedom is actuated by two servo motors. The design specifications of the robotic arm are given in Table 2. The robotic arm is compatible to work with Arduino. The real-time signal acquired is fed to MATLAB for processing and given as input to the DNN model and the model's binary output value is fed to Arduino to control the robotic arm.

Table 1 Design Specifications of Robotic Arm

\begin{tabular}{ll}
\hline & Robotic Arm \\
\hline Servo motor & Torque: $1.8 \mathrm{~kg}-\mathrm{cm}$ \\
& Weight: 9 grams \\
& Gear Type: Plastic \\
& Quantity: 3 \\
Material & Polylactic acid \\
Degrees of freedom & 2 \\
Weight & 200.0 grams \\
\hline
\end{tabular}

\section{E. Overall Design}

The workflow of MI-EEG based BCI for robotic arm control is shown in Fig. 4.

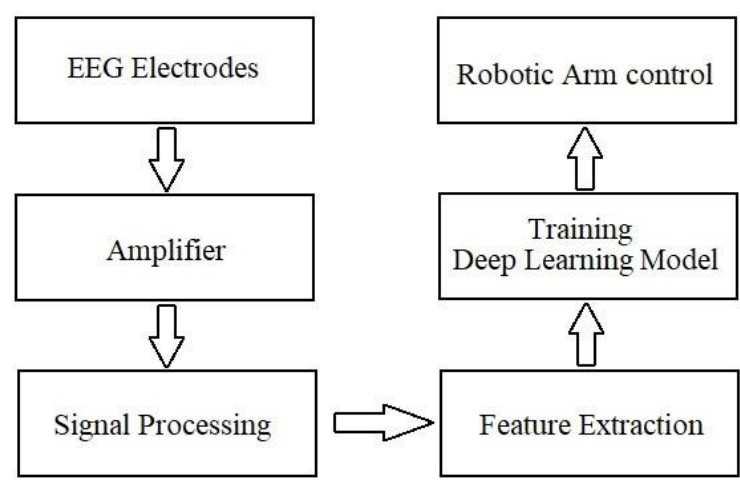

Fig. 4 Proposed system design
At first, button electrodes are used for the EEG data acquisition which is followed by signal amplification. To obtain clear and artefact-free EEG signals, signal processing is applied using the Butterworth filter and Wavelet Transform. Statistical features are extracted from the de-noised signal which is then fed to a machine learning algorithm, namely Deep Neural Network (DNN). The network is trained on the extracted features to get the optimal classification boundary. Finally, realtime data is fed into the trained DNN to obtain the classification result then the output is sent to the robotic arm, which is actuated based on the type of signal received.

\section{F. Performance Measures}

$\mathrm{K}$-Fold cross-validation is a technique used for evaluating ML models by dividing the original sample into a training set for the model to be trained and a test set for evaluation. The original sample is randomly but equally divided into subsamples of $k$ size. Out of the $\mathrm{k}$ sub-samples, $k-1$ sub-samples are used to train the model and one is used for testing the model. This process is then repeated $k$ number of times, wherein every one of the $k$ subsamples is used as the testing data exactly once. The final estimate can be obtained by taking the average of all the $\mathrm{k}$ results. The main advantage of this method is that all samples are used for training as well as validation, and each observation is used once for validation.

True Positive reflects the positive class of samples which are predicted correctly, which means 1 as 1 . False Positive reflects the positive class of samples which are predicted wrongly, giving 1 as 0 . True Negative reflects the negative class of samples which are predicted correctly, which means 0 as 0 . False Negative reflects the negative class of samples which are predicted wrongly, giving 0 as 1 .

TP, TN, FP, FN denotes true positives, true negatives, false positives, and false negatives. TP and TN are the two distinct class values that hold correct predictions and FP and FN are two distinct class values that hold incorrect predictions.

Accuracy, Precision, Recall, and F1-score are used to evaluate the DNN model and they are calculated using (1) to (4).

$$
\begin{aligned}
\text { Accuracy } & =\frac{(T P+T N)}{(T P+T N+F P+F N)} \\
\text { Precision } & =\frac{T P}{T P+F P} \\
\text { Recall } & =\frac{T P}{T P+F N} \\
\text { F1 score } & =2 * \frac{\text { Recall } * \text { Precision }}{\text { Recall }+ \text { Precision }}
\end{aligned}
$$

F1 Score is the weighted average of Precision and Recall. This is to be taken into consideration if there is an unbalanced class of sample data distribution. Accuracy will be considered when there is a balanced class of sample data distribution. 


\section{Results}

\section{A. Signal Preprocessing Results}

Data acquisition from all five subjects for each class is taken at their places at a convenient time. The waveform in red corresponds to the class "without hand movement" i.e. when the subject doesn't move the hand and the waveform in blue represents the class "with hand movement" i.e. when the subject moves the hand up which is shown in Fig. 5 a. Each recording is then processed by passing it through a bandpass filter between twelve and thirty Hertz, employing a fourth-order Butterworth filter to get rid of unwanted artefacts. It removes unwanted waveband, thereby minimizing the number of features. It has an immediate impact on reducing the execution time and decreases the use of memory, which boosts the system performance. The de-noised signal [12],[17] is then applied with the Symlet-9 wavelet transformation. It eliminates ocular physical object noise, eye blinking noise, and internal organ artefacts.

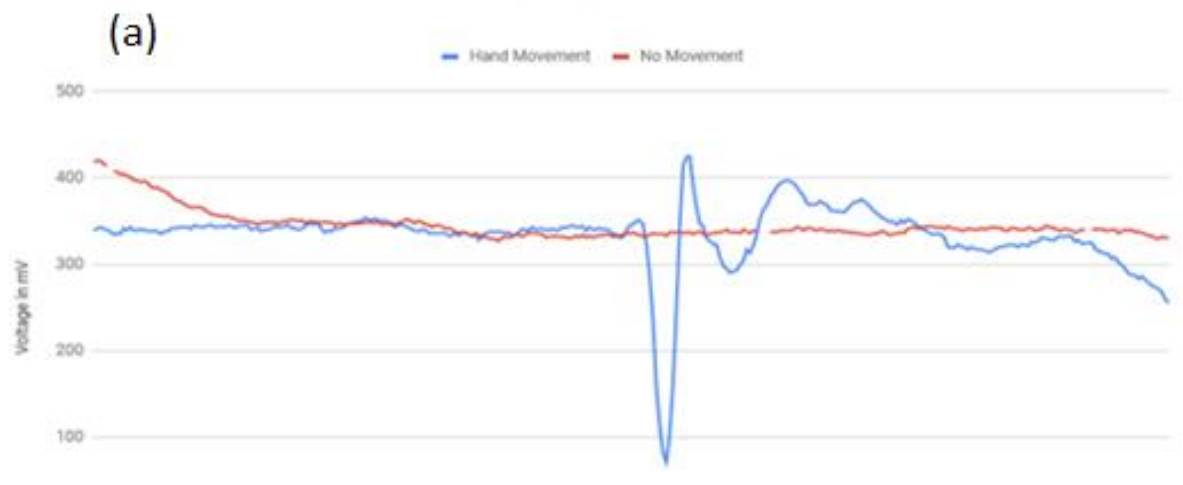

\section{(b)}
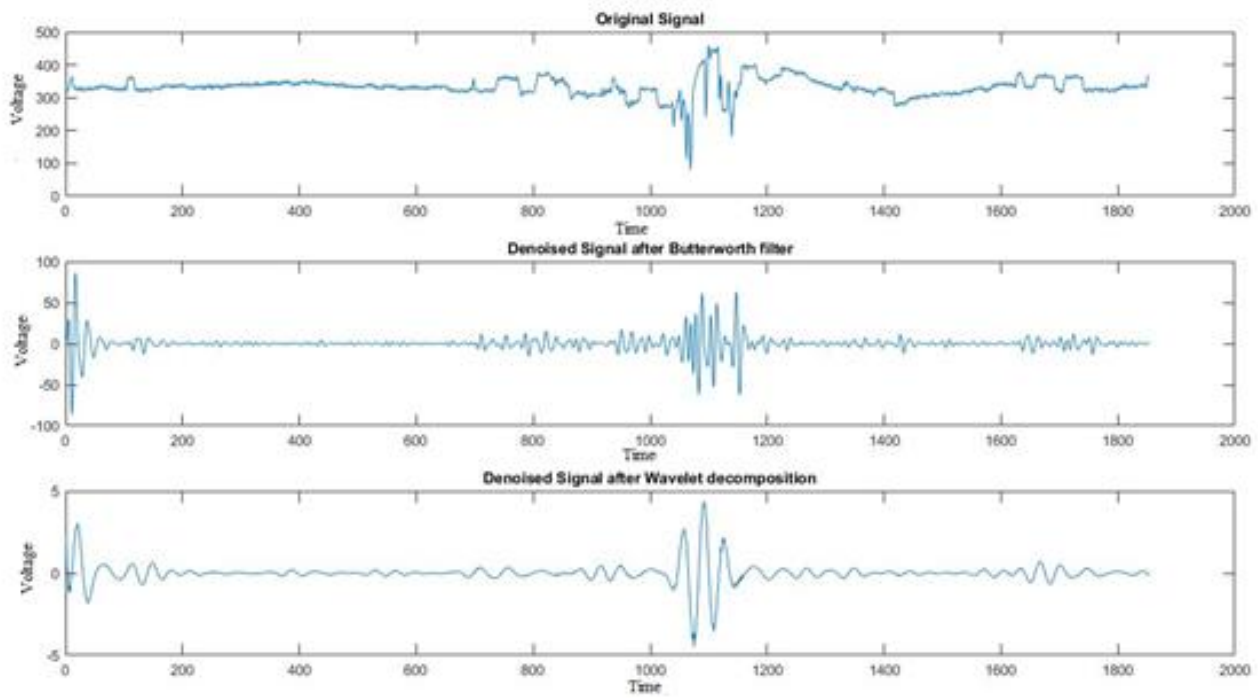

Fig. 5 EEG Waveforms (a) EEG waveform of hand movement and no hand movement Pre-processing on one sample of data. (b) raw EEG signal and pre-processed EEG signal

The preprocessing of the EEG signal is shown in Fig. 5 b. The first waveform corresponds to the raw data extracted from a human subject. The $\mathrm{x}$-axis corresponds to the number of samples acquired for a particular amount of time at a specified baud rate and the y-axis corresponds to the voltage of the signal extracted which has been amplified. The second waveform in Fig. 7 is the signal output after the raw signal has been passed through the Butterworth filter to remove initial noises [14]. The third waveform shows the final de-noised signal acquired after subjecting the Butterworth filtered signal to Wavelet Decomposition. The magnitude spectrum of Butterworth filter output is shown in Fig. 6. which confirms the signal frequency is between 12 to $30 \mathrm{~Hz}$. 


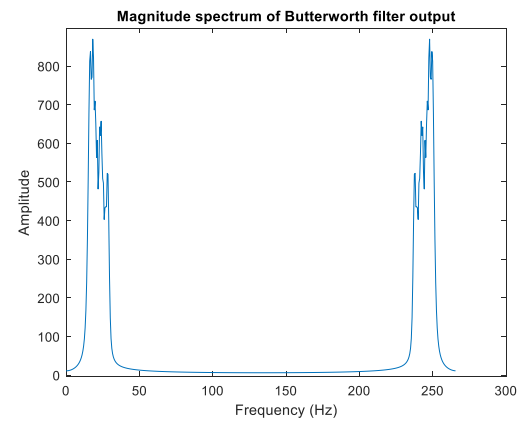

Fig. 6 Magnitude spectrum of Butterworth filter output

\section{B. Deep Neural Network-Multi-Layer Perceptron Results}

The implementation of the proposed DNN was written in python script in the Jupiter Python notebook. The handcollected dataset size of 250 was split into $80 \%$ data for training and $20 \%$ for Testing. We have employed a K-fold crossvalidation measure to validate the training accuracy.

The 14 statistical features from the feature extraction process were given as input to the DNN input layer. The network is trained with Adam optimizer and loss function as binary crossentropy. The network is trained for 200 epochs with a batch size of 5 samples. The final training accuracy obtained was $94.52 \%$ and the K-Fold Cross validated accuracy was $85.41 \%$ with $k$ as 10. The performance of DNN training and validation results are shown in Table 4.

Table 4. Training and validation performance of DNN

\begin{tabular}{cc}
\hline $\begin{array}{c}\text { Training accuracy } \\
\text { [202 samples] }\end{array}$ & $94.52 \%$ \\
\hline $\begin{array}{c}\text { K-Fold Cross validated the } \\
\text { accuracy }\end{array}$ & $83.86 \%$ \\
\hline Error rate & $5.48 \%$
\end{tabular}

The trained DNN model is tested with the test data set of 48 samples that were fed to the trained DNN classifier. Table 5 is the confusion matrix, which summarizes the test output of the DNN model used for classification. Here, out of 48 test samples, 20 correct and 0 incorrect predictions were made for the class 'No Hand Movement' which is represented by class 0 . Also, 21 correct and 7 incorrect predictions were made of class 'Hand movement' which is represented by class 1 .

Table 5 Confusion matrix of test data

\begin{tabular}{lcll}
\hline \multirow{2}{*}{ True } & \multicolumn{3}{c}{ Predicted Value } \\
\cline { 2 - 4 } Value & Class & $\mathbf{0}$ & $\mathbf{1}$ \\
\cline { 2 - 4 } & $\mathbf{0}$ & 20 & 0 \\
& $\mathbf{1}$ & 7 & 21 \\
\hline
\end{tabular}

Performance metrics such as Accuracy, Precision, Recall Sensitivity, Specificity, and F1 Score were also calculated as per the equations 1 to 4 and are listed in Table 6. Precision gives an idea about how accurate the positive predictions are. Recall Sensitivity is the coverage of actual positive samples and
Specificity is the coverage of actual negative samples. F1 score is the harmonic mean between precision and recall. It is a metric used to rate the performance of an algorithm. A $100 \%$ recall sensitivity was obtained for the class 'No Hand Movement' i.e. the trained DNN model was able to predict the class 'No Hand Movement' with a hundred percent accuracy.

Table 6 Performance metrics of DNN

\begin{tabular}{ll}
\hline Metric & Value (\%) \\
\hline Accuracy & 85.4 \\
Precision & 74 \\
Recall sensitivity & 100 \\
Specificity & 75 \\
F1 score & 85.1 \\
\hline
\end{tabular}

Table 7. Performance of ML algorithms

\begin{tabular}{cc}
\hline ML algorithm & $\begin{array}{c}\boldsymbol{K} \text {-fold cross-validation } \\
\text { Accuracy }\end{array}$ \\
\hline Logistic Regression & $72 \%$ \\
\hline Decision Tree & $73 \%$ \\
\hline SVM & $71 \%$ \\
\hline Kernel SVM & $72 \%$ \\
\hline
\end{tabular}

The binary result from the DNN model is saved as .csv file which was fed to the .m file to control an Arduino which in turn controls the robotic hand movement as shown in Fig. 7. End to end setup from signal acquisition to the robotics arm movement is shown in Fig. 7.

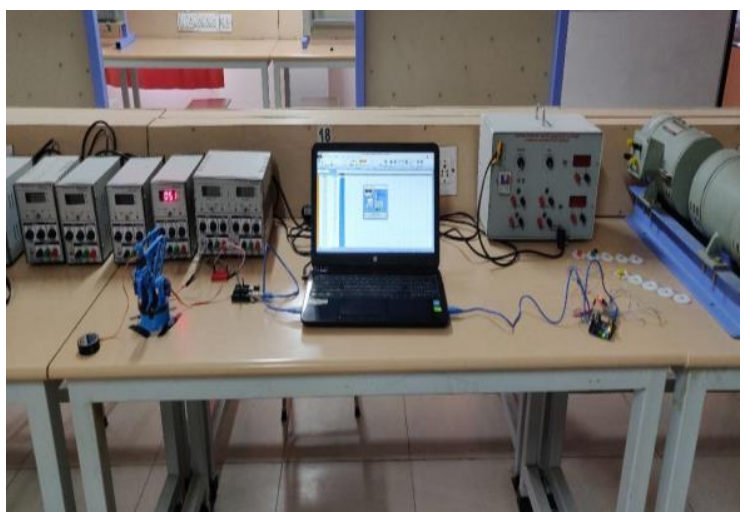

Fig.7 End to end setup for complete testing

\section{DISCUSSION}

We presented an end to end framework for robotic arm control using the EEG signal in this paper. After a thorough study with literature, we have chosen 3- channel [31] data acquisition because it removed the need for dimensionality 
reduction in our work. Among the type of EEG signal collection, we have gone with Motor Imagery (MI) EEG signal. As mentioned in the challenges, the standard data set of MI EEG for this robotic arm control is not available so we have done the collection of data from 5 healthy subjects, after getting their consent for this research, of age ranging from 20 to 60 . The raw MI EEG signal is amplified to 300 to $400 \mathrm{mV}$ using the AD8232 amplifier circuit and is further de-noised with fourth-order- Butterworth filter for power interference and Sym-9 wavelet decomposition for Ocular artefacts. In total 250 samples were collected with balanced data collection for both classes (hand movement, no-hand movement). Traditional statistical features were extracted from the pre-processed signal and applied to a custom-designed DNN-MLP classifier. Based on past work [33], this kind of architecture design has been proved to be the most efficient for classification.

The fourteen statistical features, as mentioned in the proposed work, were extracted from the de-noised signal which was fed into the DNN model. The four-layer DNN was then trained on $80 \%$ of the data and evaluated with the k-fold crossvalidation method. Next, the trained model is tested on the rest of the $20 \%$ data. Subsequently, the performance measures Accuracy, Precision, Recall Sensitivity, Specificity, and F1 Score and then displays as in Table 5. Here, we obtain the accuracy as $85.4 \%$, which is more than the value other literature reported. Even though we calculate the F1 score, for this scenario of a balanced data set, an accuracy measure will suffice for a balanced dataset. The challenges were getting the data from the subjects and the low SNR of the EEG signal. We have custom designed our signal acquisition with a simple 3electrode system based on proven literature which reduced the feature dimensions. Also, to remove the ocular artefacts, all mother wavelet basis functions were tried and it was found Sym-9 wavelet removed the artefacts effectively. We did not choose other DNN algorithms such as LSTM and CNN because they require a lot of data, due to the non-availability of standard data set available. This sets a limitation in improving the accuracy of the system using other DNN approaches. We have trained a few of the popular machine learning models such as logistic regression, Random Forest, Support Vector Machines and Decision Tree. Their classification accuracy is shown in table 8 and it was observed that the accuracy was below $75 \%$ which concludes that our proposed DNN model is superior to the ML models.

\section{CONCLUSION}

The paper presents a BCI system that used the brain's MI EEG signal associated with hand movements to control an external robotic arm. The EEG signals were extracted using a 3-channel electrode system with the AD8232 amplifier. Signal preprocessing was applied using Butterworth band-pass filter and wavelet packet decomposition technique to obtain required beta waves. Statistical features were extracted from the preprocessed signal and were then used to train an ML algorithms. Logistic regression, Random Forest, Support Vector Machines, Decision Tree and MLP-DNN were selected as the ML algorithms to classify the hand movement into two classes, 'No Hand Movement' and 'Hand Movement'. Further, Arduino and MATLAB are interfaced together to control the robotic arm based on the output predicted by the DNN model. An accuracy of $85.41 \%$ is obtained on the test set using the ANN classifier and the robotic arm is successfully actuated in the desired motion. Also, a high recall sensitivity of $100 \%$ and an F1 score of $85.1 \%$ was achieved.

Though the BCI research has been carried out for more than a decade, applying deep learning algorithm for BCI is relatively new approach. Recently, researchers focus on to develop an efficient deep learning algorithm implementation which can produce a maximum accuracy in robotic limb control. The proposed paper consists of a system to control a robotic arm mimicking a prosthetic arm using just the brain signals. Overall, this paper adds a significant contribution to the end to end realtime BCI research.

In the future, more degrees of freedom will be added to the hand movement in such a way that the robotic arm can pick up and drop an object. Also, transfer learning and a hyperparameter optimization technique like Grid Search can be explored to improve the efficacy of BCI.

\section{References}

[1] D. J. McFarland and J. R. Wolpaw, "Brain-computer interface operation of robotic and prosthetic devices," Computer (Long. Beach. Calif)., vol. 41, no. 10, pp. 52-56, 2008.

[2] J. Meng, S. Zhang, A. Bekyo, J. Olsoe, B. Baxter, and B. $\mathrm{He}$, "Noninvasive Electroencephalogram Based Control of a Robotic Arm for Reach and Grasp Tasks," Sci. Rep., vol. 6, no. November, pp. 1-15, 2016.

[3] H. A. Shedeed, M. F. Issa, and S. M. El-Sayed, "Brain EEG signal processing for controlling a robotic arm," Proc. 2013 8th Int. Conf. Comput. Eng. Syst. ICCES 2013, no. November, pp. 152-157, 2013.

[4] E. Iáñez, J. M. Azorín, A. Úbeda, J. M. Ferrández, and E. Fernández, "Mental tasks-based brainrobot interface," Rob. Auton. Syst., vol. 58, no. 12, pp. 1238-1245, 2010.

[5] C. Guan, N. Robinson, V. S. Handiru, and V. A. Prasad, "Detecting and tracking multiple directional movements in EEG based Bel," 5th Int. Winter Conf. Brain-Computer Interface, BCI 2017, pp. 44-45, 2017.

[6] J. Kronegg, G. Chanel, S. Voloshynovskiy, and T. Pun, "Eeg-based synchronized brain-computer interfaces: A model for optimizing the number of mental tasks," IEEE Trans. Neural Syst. Rehabil. Eng., vol. 15, no. 1, pp. 50-58, 2007.

[7] D. Zhang, X., Yao, L., Wang, X., Monaghan, J. and Mcalpine, "A survey on deep learning based brain computer interface: Recent advances and new frontiers.," arXiv Prepr. arXiv1905.04149., vol. 1, no. 1, pp. 1-35, 2018.

[8] C. Guger, B. Z. Allison, and N. Mrachacz-kersting, "A State-of-the-Art Summary 7," pp. 1-9.

[9] V. Podgorelec, "Analyzing EEG signals with machine learning for diagnosing alzheimer's disease," Elektron. ir Elektrotechnika, vol. 18, no. 8, pp. 61-64, 2012.

[10] T. J. Tanzi and J. Isnard, "Autonomous system for data collection: Location and mapping issues in post-disaster 
environment," Comptes Rendus Phys., vol. 20, no. 3, pp. 204$217,2019$.

[11] H. A. Akkar and F. Ali Jasim, "Optimal Mother Wavelet Function for EEG Signal Analyze Based on Packet Wavelet Transform,” Int. J. Sci. Eng. Res., vol. 8, no. 2, pp. 1222-1227, 2017.

[12] M. Balamareeswaran and D. Ebenezer, "Denoising of EEG signals using Discrete Wavelet Transform based Scalar Quantization,” Biomed. Pharmacol. J., vol. 8, no. 1, pp. 399406, 2015.

[13] A. Asadi Ghanbari, M. Nazari Kousarrizi, M. Teshnehlab, and M. Aliyari, "Wavelet and Hilbert transform-based brain computer interface," 2009 Int. Conf. Adv. Comput. Tools Eng. Appl. ACTEA 2009, pp. 438-442, 2009.

[14] G. \& V. K. J. \& A. S. Kaushal, "Removal of Power Line Interference from EEG using Wavelet-ICA,” no. Icaet, pp. 2931, 2015.

[15] V. Vanitha and P. Krishnan, "Time-frequency analysis of EEG for improved classification of emotion," Int. J. Biomed. Eng. Technol., vol. 23, no. 2-4, pp. 191-212, 2017.

[16] L. Sun and Z. R. Feng, "Classification of imagery motor EEG data with wavelet denoising and features selection," Int. Conf. Wavelet Anal. Pattern Recognit., vol. 2016-November, pp. 184-188, 2016.

[17] G. B. Bernardi, T. C. Pimenta, and R. L. Moreno, "A Simplified Tool for Testing of Feature Selection and Classification Algorithms in Motor Imagery of Right and Left Hands of EEG Signals," 2019 IEEE 10th Lat. Am. Symp. Circuits Syst. LASCAS 2019 - Proc., pp. 197-200, 2019.

[18] K. Mahajan, "Classification of EEG using PCA, ICA and Neural Network," Int. J. Eng. Adv. Technol., vol. 1, no. 1, pp. 80-83, 2011.

[19] I. Omerhodzic, S. Avdakovic, A. Nuhanovic, and K. Dizdarevic, "Energy distribution of EEG signals: EEG signal wavelet-neural network classifier," World Acad. Sci. Eng. Technol., vol. 37, pp. 1240-1245, 2010.

[20] and G. D. Rami N. Khushaba, Sarath Kodagoda, Sara Lal, "Driver Drowsiness Classification Using Fuzzy Wavelet Packet Based Feature Extraction Algorithm," IEEE Trans. Biomed. Eng., vol. 6, no. 1, pp. 1-11, 2010.

[21] M. Mumtaz, M. Afzal, and A. Mushtaq, "Sensorimotor Cortex EEG signal classification using Hidden Markov Models and Wavelet Decomposition," 2018 IEEE Int. Symp. Signal Process. Inf. Technol. ISSPIT 2018, pp. 375-381, 2019.

[22] S. S. Devi, "Analysis of Eeg for Motor Imagery Based Classification of Hand Activities," vol. 2, no. 3, pp. 11-22, 2015.

[23] A. S. Al-Fahoum and A. A. Al-Fraihat, "Methods of EEG Signal Features Extraction Using Linear Analysis in Frequency and Time-Frequency Domains," ISRN Neurosci., vol. 2014, pp. 1-7, 2014.

[24] E. Parvinnia, M. Sabeti, M. Zolghadri Jahromi, and R. Boostani, "Classification of EEG Signals using adaptive weighted distance nearest neighbor algorithm," J. King Saud Univ. - Comput. Inf. Sci., vol. 26, no. 1, pp. 1-6, 2014.

[25] S. U. Amin, M. Alsulaiman, G. Muhammad, M. A. Bencherif, and M. S. Hossain, "Multilevel Weighted Feature Fusion Using Convolutional Neural Networks for EEG Motor Imagery Classification," IEEE Access, vol. 7, pp. 18940$18950,2019$.
[26] T. H. Shovon, Z. Al Nazi, S. Dash, and M. F. Hossain, "Classification of motor imagery EEG signals with multi-input convolutional neural network by augmenting STFT," 2019 5th Int. Conf. Adv. Electr. Eng. ICAEE 2019, no. August, pp. 398403, 2019.

[27] N. Robinson, S. Lee and C. Guan, "EEG Representation in Deep Convolutional Neural Networks for Classification of Motor Imagery," 2019 IEEE International Conference on Systems, Man and Cybernetics (SMC), Bari, Italy, 2019, pp. 1322-1326, doi: 10.1109/SMC.2019.8914184.

[28] J. M. Williams, "Deep Learning and Transfer Learning in the Classification of EEG Signals," Deep Learn. Transf. Learn. Classif. EEG Signals, pp. 1-82, 2017.

[29] C. T. F. S. W. Zhang, "DEEP TRANSFER LEARNING FOR EEG-BASED BRAIN COMPUTER INTERFACE," ICASSP, pp. 916-920, 2018.

[30] H. Cho, M. Ahn, S. Ahn, M. Kwon, and S. C. Jun, "EEG datasets for motor imagery brain-computer interface," Gigascience, vol. 6, no. 7, pp. 1-8, 2017.

[31] X. G. and S. G. Y. Wang, B. Hong, "Design of electrode layout for motor imagery based brain-computer interface," Electron. Lett., vol. 43, no. 10, pp. 10-11, 2007.

[32] Martina Zabcikova, Zuzana Koudelkova, Roman Jasek, Investigation of Emotiv Epoc+ Headset Usability by Measuring Various Stimuli, WSEAS Transactions on Applied and Theoretical Mechanics, pp. 184-191, Volume 14, 2019.

[33] M. A. Haque, J. S. Rani Alex, and N. Venkatesan, "Evaluation of Modified Deep Neural Network Architecture Performance for Speech Recognition," Int. Conf. Intell. Adv. Syst. ICIAS 2018, pp. 1-5, 2018.

[34] Robotic Arm with gripper Diy kit https://www.amazon.in/Ayasa-Electronics-Robotic-IncludedIncluded/dp/B077YMCBWW accessed 25/8/2020

\section{Creative Commons Attribution License 4.0 (Attribution 4.0 International, CC BY 4.0)}

This article is published under the terms of the Creative Commons Attribution License 4.0 https://creativecommons.org/licenses/by/4.0/deed.en_US 